\title{
Informes
}

Reunión-diálogo sobre el Programa Nacional de Población $1995-2000$ y sus implicaciones

Beatriz Figueroa*

José Luis Lezama*

Manuel Ordorica

La reunión que ahora se reseña fue promovida por el Centro Tepoztlán (que se ha caracterizado por la organización de reuniones-diálogo que analizan temas de actualidad) y contó con la participación de casi cuarenta personas. La sesión del 28 de octubre de 1995 tuvo como objetivo reunir a un grupo multidisciplinario para reflexionar sobre el Programa Nacional de Población 1995-2000 (Conapo, 1995), de acuerdo con los siguientes temas generales: las metas demográficas que establece dicho programa y la posibilidad técnica de cumplirlas; el papel específico de los programas de cobertura de la planificación familiar que deben aplicarse o desarrollarse, considerando la infraestructura y los procedimientos ya existentes o en curso de adaptación; los requisitos complementarios de una política integral de población, en particular lo que se refiere al estatus de la mujer, la salud reproductiva, la participación en la fuerza de trabajo, la educación y la comunicación social; la perspectiva de evolución de la población mexicana y de su estructura más allá del año 2005; la relación entre las recomendaciones propuestas en la Conferencia de las Naciones Unidas sobre Población y Desarrollo de El Cairo y la política poblacional mexicana; y por último, el significado de la perspectiva demográfica mexicana frente a la nueva estrategia de desarrollo del país.

\section{Sesión matutina}

La sesión dio inicio con las palabras de bienvenida de Víctor L. Urquidi, seguidas de una breve presentación de los objetivos de la reunión hecha por el profesor Gustavo Cabrera de El Colegio de México y por el doctor Jorge Martínez Manautou de la Academia Mexicana de Investigación en Demografía Médica, ambos organizadores del seminario. xico.

* Centro de Estudios Demográficos y de Desarrollo Urbano de El Colegio de Mé- 
En primer término, Gustavo Cabrera destacó que el Programa Nacional de Población (PNP) fue elaborado adecuadamente y cuenta con una alta calidad profesional, no obstante, podría tener limitaciones operativas por el desorden económico y financiero que prevalece en la actualidad, y por las circunstancias políticas por las que atraviesa el país. El Programa plantea la prioridad de incidir en la circularidad entre pobreza y rezago demográfico, además de que señala la existencia de un desempleo abierto con tasas elevadas o de las más elevadas en los últimos 50 años, así como la inflación y el deterioro de las condiciones sociales elementales de la población. Ambos retos se enfrentan en sentidos opuestos a los objetivos.

El profesor Cabrera hizo hincapié en otros aspectos importantes, como por ejemplo, que el programa se ubica en un tiempo importante de la transición demográfica; que se deben remontar inercias pasadas para alcanzar los niveles de reemplazo de la fecundidad; se pretende descentralizar las acciones y los recursos que favorezcan una mayor efectividad para encarar los rezagos demográficos regionales; los Consejos Estatales y aun los municipales requieren de nuevas estrategias en su quehacer; se requiere fortalecer la cultura demográfica a fin de crear conciencia de los fenómenos poblacionales en la sociedad, la familia y los individuos; dicha conciencia también debe estar presente entre políticos y funcionarios. En fin, el programa tiene condiciones favorables de tipo programático, de enfoque y de intenciones.

El doctor Jorge Martínez Manautou destacó que el Programa Nacional de Población presenta las metas que deberán alcanzarse en materia de planificación familiar. En este sentido, la parte programática es la menos complicada: lo que preocupa es cómo, quién y a partir de qué se van a operar las acciones correspondientes. En esta reunión, añadió, es importante discutir las estrategias diseñadas por Conapo y por las instituciones del sector salud para enfrentar las dificultades que se aprecian durante el periodo 1995-2000, el último del siglo que está por terminar.

Martínez Manautou insistió en que la oportunidad de dialogar con los responsables del Programa Nacional de Población en un ambiente académico, es una clara intención de apoyarlo. Por ello, es recomendable que este diálogo continúe y se convierta en un mecanismo de evaluación del Programa, que se evite su aislamiento ya que en el pasado reciente este aislamiento resultó contraproducente, además de que se discuta la participación del sector privado, tan disminuida en los últimos años. 
Los ponentes de la sesión de la mañana fueron el doctor José Gómez de León, secretario general del Consejo Nacional de Población y el doctor Gregorio Pérez Palacios, director general de Salud Reproductiva de la Secretaría de Salud, el doctor Alejandro Aguirre, profesor-investigador de El Colegio de México y el maestro Agustín Porras, de la Universidad Autónoma Metropolitana.

\section{Ponentes}

El doctor José Gómez de León destacó, en primer término, que la política de población tiene un marco de definición respetuoso de los derechos y libertades individuales así como de las parejas. El Programa Nacional de Población en su diagnóstico menciona los aspectos más destacados y críticos a los que se enfrenta la política de población; al señalar las principales cifras demográficas, Gómez de León mencionó que la población de México llegará en el año 2000 a 100 millones de habitantes aproximadamente, para alcanzar 130 millones en el año 2030.

En relación con el comportamiento reproductivo, se presentaron los diferenciales de fecundidad entre distintos grupos sociales y regiones del país, entre los que se destacan los siguientes: en 1990, las mujeres sin instrucción escolar tenían, en promedio, 5.6 hijos, mientras que las que tenían secundaria completa o más tenían sólo 2.4 hijos; las mujeres que habitaban en localidades rurales tenían cerca de 5 hijos y las que residían en áreas metropolitanas 2.6 hijos. En la fecundidad por entidad federativa también se observan diferencias importantes: en Chiapas, Guerrero y Oaxaca las mujeres tuvieron 4.5 hijos en el periodo 1987-1991, mientras que en el Distrito Federal tuvieron 2.3 y en Nuevo León 2.5 .

La práctica anticonceptiva se ha extendido durante las últimas dos décadas. En los años 1974-1976, la prevalencia de uso de métodos anticonceptivos, se estima en $30 \%$ de las mujeres casadas y unidas en edad fértil. Esta cobertura se incrementó a $48 \%$ en 1982, lo que significa un aumento anual cercano a 3 puntos. En los siguientes cinco años, de 1983 a 1987, se observa una marcada desaceleración en el ritmo de crecimiento anual de los niveles de uso, con aumento promedio de un punto por año, el cual se recupera en el quinquenio de 1988-1992, donde el incremento anual de prevalencia fue de dos puntos, hasta llegar a 63\% en 1992. 
El uso de métodos anticonceptivos por entidad federativa muestra también importantes diferencias: en 1992 en Chiapas, Guerrero y Oaxa$\mathrm{ca}$, las mujeres en edad fértil registran una cobertura de $50 \%$, mientras que en el Distrito Federal, Baja California y Nuevo León fue de cerca de $80 \%$. De igual manera, de acuerdo al grado de escolaridad, la cobertura fue de $38 \%$ de mujeres en edad fértil sin instrucción frente a $74 \%$ de aquellas que tenían secundaria completa y más años de estudio.

En la práctica anticonceptiva en México se observó durante 1992 el uso de métodos modernos: $88 \%$ de las usuarias empleaba un método hormonal, un dispositivo intrauterino o un método quirúrgico. En 1992 se observa que la oclusión tubaria bilateral es el tipo de método más importante $(43.3 \%)$, seguido del dispositivo intrauterino $(17.7 \%)$ y luego de los orales $(15.3 \%)$; los métodos tradicionales representaban $12 \%$. El método que ha ganado más importancia es la esterilización femenina, y por el contrario, el uso de las pastillas ha disminuido notablemente.

El aumento en el suministro de los anticonceptivos del sector público es muy importante; gran parte de su cobertura se observa en el Instituto Mexicano del Seguro Social (IMss), que en 1992 atendió a dos tercios de las usuarias del sector público. El imss registró un cambio de usuarias de anticonceptivos de 30 comparado con $40 \%$ entre 1979 y 1992. Por otra parte, la Secretaría de Salubridad y Asistencia se mantuvo en $15 \%$ aproximadamente. Las farmacias tenían en 1979 $30 \%$ de usuarias de anticonceptivos y en 1992 se observó un descenso hasta llegar a una cifra inferior a 20 por ciento.

Asimismo, se analizó el número ideal de hijos, el cual es diferente según lugar de residencia y escolaridad. Las mujeres que en 1987 residían en áreas rurales manifestaban un número medio de familia deseado de 4.3 hijos, mientras que las mujeres residentes en áreas urbanas deseaban 3.3 hijos. Al analizar la variable escolaridad, se observa que las mujeres que habían cursado secundaria o más deseaban 2.5 hijos, mientras que aquéllas sin instrucción deseaban 4.9 hijos.

Por último, se presentaron las metas demográficas que el PNP plantea; una de ellas, es que la tasa de crecimiento natural sea de $1.75 \%$ en el año 2000 y $1.45 \%$ en el 2005 . Esta dinámica supone tasas globales de fecundidad de 2.4 y 2.1 hijos por mujer, respectivamente. El nivel de fecundidad indicado para el año 2005 equivale al del reemplazo intergeneracional.

A fin de lograr la tasa de fecundidad programada se requiere impulsar el programa de planificación familiar de forma tal que la pre- 
valencia en el uso de métodos anticonceptivos de mujeres unidas en edad fértil ascienda a $70.2 \%$ en el año 2000 y a $73.3 \%$ en el año 2005 . Con estas metas en las prácticas anticonceptivas, el número de usuarias será, para esos años, de 12.6 y 14.8 millones respectivamente. Esto implicaría mantener, por un lado, la cobertura anticonceptiva de muijeres unidas en las áreas urbanas, que ya es mayor a $70 \%$; y por otro, elevar dicha cobertura en las áreas rurales de $44 \%$ en 1992 a poco más de $57 \%$ en el año 2000.

El doctor Gregorio Pérez-Palacios señaló en primer lugar que la planificación familiar es uno de los pilares más importantes para alcanzar las metas de impacto demográfico, así como las propias en materia de salud. Señaló que en el Programa de Reforma del Sector Salud se ratifica la meta de reducir la tasa global de fecundidad de 2.9 hijos por mujer en 1994 a 2.4 hijos a finales del año 2000. Se mencionó también la meta de cobertura para el año 2000 , de $70.2 \%$ de usuarias de métodos anticonceptivos en relación con las mujeres unidas en edad fértil. Para alcanzar este nivel de cobertura se requerirá contar con 12.6 millones de usuarias. La cobertura de anticonceptivos debería incrementarse de una prevalencia de $64 \%$ para 1994 a $70 \%$ para fines del presente siglo.

Otras metas de cobertura en materia de planificación familiar que han sido incorporadas al Programa de Reforma del Sector Salud son: proporcionar información suficiente, veraz y oportuna a toda la población sobre los conceptos y servicios de salud reproductiva y de planificación familiar; duplicar para el año 2000 el número de procedimientos de vasectomía sin bisturí, como un método anticonceptivo permanente que ha presentado una fuerte demanda y por último, proporcionar información sobre planificación familiar a todas las mujjeres durante el embarazo y después de un hecho obstétrico, es decir, posparto, poscesárea y posaborto, con el fin de incrementar la cobertura de anticoncepción a $70 \%$ en este grupo de población.

El Grupo Interinstitucional de Salud Reproductiva ha implementado un conjunto de estrategias lo suficientemente flexibles para poder atender las necesidades, requerimientos y características particulares de los grupos de población que son apoyados por las distintas instituciones.

Una estrategia fundamental es el incremento de la oferta de servicios de planificación familiar; para mejorar la calidad de los servicios, se ha ampliado la gama de métodos anticonceptivos modernos, seguros, eficaces y aceptables y se han fortalecido las acciones dirigí- 
das a los grupos vulnerables. Entre los métodos de regulación de la fertilidad de reciente incorporación está el cyclofem, un anticonceptivo inyectable de administración mensual, de gran efectividad, con efectos colaterales mínimos y con bajo costo. Se ha incorporado el uso de los implantes subdérmicos anticonceptivos, que es un método de acción prolongada con indicaciones de uso muy puntuales.

Un componente esencial del programa de salud reproductiva es la diversificación y fortalecimiento de las estrategias de información, educación y comunicación para transmitir mensajes de los servicios de planificación familiar que promuevan la adopción de actitudes y prácticas de paternidad responsable, que favorezcan la postergación del primer nacimiento y el espaciamiento de los hijos, así como la terminación temprana de la reproducción. Para extender el uso de métodos modernos en el área rural se capacita a supervisoras de auxiliares de salud en la promoción, consejería, inserción y remoción del dispositivo intrauterino.

Se ha realizado un ejercicio para estimar la combinación en el uso de métodos anticonceptivos que se registrará en México a finales del siglo: se prevé un ligero aumento en la participación relativa del dispositivo intrauterino, de los hormonales inyectables y de la vasectomía sin bisturí. La oclusión tubaria bilateral seguirá siendo el método anticonceptivo más utilizado por la población mexicana: se espera que para el año 2000, 45 de cada 100 usuarias hayan recurrido a este método.

La salud reproductiva de los adolescentes constituye un reto en las políticas de salud, de educación y de bienestar social. Para ello el objetivo es proporcionar información y servicios en salud reproductiva dentro de un marco de atención integral a la salud que fomente y modifique actitudes y conductas responsables, permitiendo que los adolescentes asuman su sexualidad de manera autónoma, consciente $y$ sin riesgos.

Como metas de cobertura se ha planteado proporcionar información suficiente, veraz y oportuna sobre salud reproductiva a todos los adolescentes, con el fin de propiciar el autocuidado y prevenir embarazos no deseados, abortos y enfermedades de transmisión sexual. Las metas de impacto que se obtendrán al fortalecer la atención de la salud reproductiva de los adolescentes son postergar la edad al nacimiento del primer hijo, reducir $50 \%$ los embarazos no deseados en mujeres menores de 20 años y reducir la incidencia de enfermedades de transmisión sexual en la población adolescente. 
En nuestro país se ha presentado en los últimos años un resurgimiento de las enfermedades de transmisión sexual además de las infecciones tradicionales y del SIDA. Las metas de cobertura son lograr que todo el personal del primer nivel de la atención conozca y aplique los criterios y la normatividad para la prevención, diagnóstico temprano, tratamiento o referencia de las enfermedades de transmisión sexual, otorgar el tratamiento oportuno y adecuado a todos los casos diagnosticados y a sus contactos e informar a la población en general acerca de las medidas preventivas y los sitios de atención, así como advertirla sobre las secuelas y complicaciones tempranas y tardías de las enfermedades de transmisión sexual.

Pérez Palacios destacó finalmente que todos los programas y acciones de salud reproductiva se realizan en el nuevo programa con particular atención a la perspectiva de género, facilitando la emancipación y respetando los derechos de la mujer, con el fin de propiciar una mayor equidad en las relaciones entre los géneros.

En relación con las metas demográficas, el doctor Alejandro Aguirre destacó que las metas propuestas en el PNP, sobre crecimiento natural de la población, son más modestas que en el pasado. Para fundamentar esta afirmación, citó la página $60 \mathrm{del} \mathrm{PNP} \mathrm{y} \mathrm{comparó} \mathrm{las}$ metas actuales con las de programas anteriores. El Programa dice:

la meta en materia de crecimiento demográfico para el país es alcanzar una tasa de crecimiento natural de $1.75 \%$ en el año 2000 y $1.45 \%$ en el año 2005. Esta dinámica demográfica supone tasas globales de fecundidad de 2.4 y 2.1 hijos por mujer, respectivamente. El nivel de fecundidad indicado para el año 2005 equivale a la consecución del reemplazo intergeneracional, que es la meta que gobierna las estrategias programáticas en la materia.

En cambio, en 1977 se estableció como meta una tasa de crecimiento natural (TCN) de $1 \%$ para el año 2000. La viabilidad de este propósito ha sido ampliamente discutida, ya que para cumplirla se tendría que llegar a una tasa neta de reproducción (TNR) por debajo del reemplazo, equivalente a: TNR= 0.876 durante el último lustro del siglo y con una reducción aún mayor para fechas posteriores ( $\mathrm{TNR}=0.667)$.

De acuerdo con las tendencias observadas se ha visto que es imposible llegar a un nivel del reemplazo para el año 2000 o tener una cobertura en materia de prevalencia anticonceptiva de $100 \%$. Si bien es cierto que se alcanzó la meta de TNC para 1982 (2.5), a partir de esa fecha las metas propuestas no han sido logradas: TCN de $1.8 \%$ en 1988 
y de $1.3 \%$ en 1994. En el PNP se estima que la TCN correspondiente a 1995 es de 2.05, que como se puede observar, es muy superior a las metas fijadas en 1977.

En el PNP actual, la meta de la TCN para el año 2000 es $1.7 \%$, que es tan sólo un poco menor que la que originalmente se programó para 1988 , de $1.8 \%$. La señalada para el año 2005 es $1.45 \%$, mayor inclusive que la anteriormente propuesta para el año 2000, de $1 \%$. Asimismo, las metas en el PNP para las tasas netas de reproducción fueron hechas con mesura, porque es hasta el año 2005 cuando se supone que la tasa neta de reproducción sea menor que el reemplazo. En cuanto a la práctica anticonceptiva, las metas propuestas sobre cobertura están dentro de lo posible. Se estima que en el año $2000,70.2 \%$ de las mujeres unidas en edad fértil estarán haciendo uso de métodos anticonceptivos, y para el año 2005, esta cifra ascenderá a 73.3 por ciento.

No obstante que las metas fijadas tanto en materia de crecimiento natural como de anticoncepción son planteamientos viables en el PNP, el doctor Aguirre indicó que en este programa hace falta que se especifique el tipo de método anticonceptivo que se va a utilizar para alcanzar las metas sobre fecundidad.

Otra omisión en el pNp es que no se señalan las metas de crecimiento natural o de fecundidad según región o por ámbitos menores como las entidades, sólo se señalan aquéllas para el total del país. Por ejemplo, para el año 2005 si se llevara a cabo una reducción proporcional uniforme en la TGF, sería del orden de 3.0 hijos por mujer en el campo y de 1.8 en las ciudades. Así, la fecundidad en el medio urbano tendría que estar por debajo de los niveles de reemplazo (esta tasa sería similar a la actual de Cuba y menor que la que se registra en Estados Unidos, de 2.1).

Por lo anterior, el doctor Aguirre recomendó que el PNP incluya metas según el tamaño de la localidad y que se evalúe su factibilidad. Lo mismo se puede decir respecto de las entidades federativas dadas las disparidades que existen. Como ejemplo señaló el caso de Chiapas, que para el año 2000 tendría que alcanzar una tasa global de fecundidad de 3.19 y en el año 2005 de 2.79 , pero si lo comparamos con el Distrito Federal, esta tasa global de fecundidad tendría que ser de 1.5 hijos por mujer al final del siglo y de 1.3 hijos cinco años después. Estos datos indican que no es factible que la brecha se cierre por completo entre las diversas entidades y resulta difícil pensar que, por ejemplo en Chiapas, se pudiera llegar a una fecundidad a nivel de 
reemplazo en 2005, cuando en el censo de 1990 se encontró una tasa de analfabetismo femenino de 37.5 por ciento.

El doctor Aguirre recomendó una especial atención a los métodos que se utilizarán para llegar a las metas propuestas. El método que más impacto tiene sobre el descenso de la fecundidad tanto por su virtual infalibilidad como por su irreversibilidad es la esterilización femenina. Ésta ha aumentado con el tiempo y se ha convertido en el método de regulación de la fecundidad más recurrido; la tendencia es que siga aumentando y su efecto en la fecundidad será entonces innegable. Sin embargo, si se le sigue utilizando como se le ha empleado hasta ahora no se asegura que se cumplan las metas: en la Encuesta Nacional de la Dinámica Demográfica (Enadid) 1992, se encontró que más de $80 \%$ de las mujeres esterilizadas tenía tres o más hijos vivos y que el promedio de hijos vivos de todas las mujeres esterilizadas era de cuatro.

Por otro lado, habría que considerar otro elemento: el referente a los proveedores de servicios en materia anticonceptiva. Los datos más recientes que se tienen en esta materia, proporcionados por la Enadid de 1992, indican que dos tercios de las usuarias de métodos anticonceptivos obtienen estos últimos del gobierno, siendo el ımss el principal proveedor con $40 \%$. La actual crisis financiera ha obligado a hacer recortes presupuestales por lo que al sector público podrían faltarle los recursos necesarios aun para mantener las coberturas de los años recientes.

Por otra parte debemos considerar que en lo que va del año, el principal proveedor (el imss), ha visto reducido su número de asegurados. Aunque los servicios de planificación familiar se ofrecen también a la población abierta, parte de ésta, por diversas razones no accede a ellos. Otro problema que se debe afrontar es la reducción de la obtención de anticonceptivos en las farmacias, las cuales han visto reducidas sus ventas durante 1995 debido a la crisis económica.

Una ausencia importante en el PNP en la sección de metas y perspectivas es la omisión de aquellas que se refieren a la mortalidad y a la migración, ambas componentes de igual importancia en la dinámica demográfica.

Las reflexiones del maestro Agustín Porras se centraron en torno de la eficiencia y cobertura de los programas de planificación familiar en México en el periodo 1970-1994. Inició su intervención recordando que a partir de 1977 se instrumentó el Plan Nacional de Planificación Familiar en el marco de las políticas de población. Este progra- 
ma tenía como objetivo aumentar el uso de anticonceptivos entre las mujeres casadas o unidas en edad fértil, para que con ello pudieran tomar sus decisiones sobre el número y espaciamiento de los hijos. El fin ulterior de este programa era reducir los niveles de fecundidad de la población, de manera que ésta se ajustara a las metas de crecimiento demográfico que había establecido el Consejo Nacional de Población. La responsabilidad de la puesta en marcha de este programa correspondió al sector salud.

Para efectuar el análisis correspondiente, el maestro Porras analizó tres periodos: 1976-1982; 1982-1987 y 1987-1992. Para cada periodo consignó las cifras sobre el porcentaje de mujeres unidas usuarias de métodos anticonceptivos, o tasa de prevalencia, para el total del país, para el ámbito rural y el urbano y según algunas características socioeconómicas, con objeto de observar las diferencias existentes. A su vez, relacionó los cambios ocurridos en el nivel de fecundidad (medido por medio de la tasa global de fecundidad, TGF) con los ocurridos en el porcentaje de usuarias de anticonceptivos, respecto del total de la población de mujeres unidas.

Considera que el desempeño del programa en el periodo 19761982 fue muy eficiente, pues la tasa de prevalencia en el uso de métodos anticonceptivos se incrementó a un ritmo anual de $9.65 \%$. La proporción de mujeres unidas usuarias, creció de $30.2 \%$ a $47.70 \%$. Asoció este hecho al descenso de la TGF de 5.49 hijos por mujer a 4.36, o sea, una caída de la TGF de 20\%. El periodo 1982-1987 lo evaluó como de baja eficiencia, ya que la prevalencia aumentó a un ritmo anual de $2.1 \%$, que representa cerca de una cuarta parte del ritmo del periodo anterior. El número de mujeres usuarias pasó de $47.6 \%$ a sólo $52.7 \%$. El ritmo en la disminución de la fecundidad también se desaceleró, pues sólo disminuyó $10.7 \%$ en este periodo. La TGF estimada para 1987 fue de 3.89 hijos por mujer.

En el periodo 1987-1992 hubo un repunte en la eficiencia del Programa: la tasa de prevalencia aumentó a un ritmo anual de $3.95 \%$, que aunque podemos pensar que no alcanzó la eficiencia del periodo 1976-1982, sí presentó una ligera recuperación; la proporción de usuarias en 1992 fue $63.1 \%$; la fecundidad en este periodo se redujo $16.7 \%$ y la TGF estimada fue de 3.24 hijos por mujer.

No obstante estos logros en el ámbito nacional, los niveles de fecundidad y las tasas de prevalencia en el uso de métodos anticonceptivos alcanzados hasta 1992 presentan diferencias notables según la estructura social mexicana. Si se considera la estratificación social de 
la población sólo mediante la variable urbano-rural, se tiene que en 1976 las tasas de prevalencia eran de $41.1 \%$ para la población urbana y $16.5 \%$ para la rural. En 1992 ambos porcentajes fueron mayores: 70.1 y $46.7 \%$, respectivamente. Si bien las diferencias entre el ámbito rural y urbano han disminuido, la brecha entre éstas es todavía de consideración. El periodo de menor eficiencia, como ya se había señalado, fue 1982-1987 en el que para las zonas rurales el ritmo de crecimiento anual fue sólo de $2.65 \%$, la mitad del ritmo de crecimiento observado en el periodo anterior. En los años 1987-1992 se logró incrementar la eficiencia, con una tasa de incremento anual de $7.45 \%$ en las zonas rurales.

En relación con la escolaridad, las diferencias son notables. En el medio rural, durante 1992 sólo $27.9 \%$ de las mujeres sin algún grado de escolaridad usaban anticonceptivos, mientras que en el medio urbano este porcentaje fue de $51.4 \%$. El maestro Porras atribuye este diferencial al acceso diferenciado que tiene la población a los servicios de salud.

En el periodo 1982-1987, el desempeño del programa de planificación familiar en el medio rural fue muy bajo en los estratos sociales de baja escolaridad, comparado con el periodo 1987-1992. Para el estrato sin escolaridad, el ritmo de crecimiento anual en el periodo 1982-1987 fue cero, es decir, no aumentó la prevalencia; mientras que el programa hizo crecer la prevalencia a un ritmo anual de $13.6 \%$ durante el periodo siguiente (1987-1992). En el medio urbano, la prevalencia entre el grupo de mujeres sin escolaridad aumentó a un ritmo anual de $10.59 \%$ en el mismo periodo de 1987-1992.

Sin duda, el bajo desempeño del programa de planificación familiar en el sexenio 1982-1987 contribuyó a mantener las diferencias urbano-rurales y según estratos socio-económicos, en los niveles de fecundidad así como en las tasas de prevalencia en el uso de métodos anticonceptivos por lo que se faltó notablemente al cumplimiento de las metas que se habían programado en el Plan Nacional de Planificación Familiar puesto en marcha en 1977.

Para finalizar su intervención, el maestro Porras presentó un analisis de regresión en el que asoció la TGF con las tasas de prevalencia de uso de anticonceptivos y el producto per cápita de las diversas entidades. La correlación encontrada entre las tasas globales de fecundidad estimadas para el periodo 1987-1991 y la tasa de prevalencia estimada para 1992 fue de 0.86 , lo cual indica que en México, durante los últimos 18 años, el aumento en el uso de métodos anticonceptivos explica 
$75 \%$ de la disminución en los niveles de fecundidad. Como hemos señalado antes, el aumento en el uso de estos métodos se explica, en gran medida, por el desempeño eficiente de los programas de planificación familiar en los periodos de 1976-1982 y de 1987-1992.

La correlación encontrada entre las tasas globales de fecundidad y los niveles de bienestar (INEGI, 1994) fue de 0.68, lo cual es indicativo de que los niveles de fecundidad en las entidades federativas se explican en un $47 \%$ por sus niveles de bienestar (INEGI, 1994); es decir, otras variables están actuando como por ejemplo, los niveles de pobreza y la falta de acceso de la población a los sistemas de salud pública.

\section{Discusión general}

En la discusión general se presentaron diversos comentarios, los cuales se pueden resumir de la siguiente manera:

1) La importancia de establecer programas demográficos para la población indígena, en que se fortalezca a la familia y se consideren los aspectos culturales, el papel de la mujer, la distribución de la población, etc., de estos grupos, con el fin de que el Programa Nacional de Población no se restrinja exclusivamente al tema relativo al elevado crecimiento de la población. De ahí la importancia de vincular lo demográfico con los otros programas socioeconómicos.

2) La conveniencia de crear una mayor conciencia en las familias y en la sociedad sobre las consecuencias del acelerado ritmo de aumento demográfico y sus relaciones con el desarrollo económico. En este sentido, se mencionó la importancia de fortalecer la educación en materia de población de los adolescentes y los ninos en edad preescolar y primaria. Para ello hay que crear una cultura de inversión en capital humano.

3) Lo ambicioso del programa de salud reproductiva, con metas tan específicas, por lo que resulta importante pasar de la planeación a la acción. Parecería que hace falta trabajar más el paso del enfoque tradicional de planificación familiar al de salud reproductiva y otros temas relacionados como son la sexualidad, las relaciones de pareja y la educación del hombre en la atención a los hijos.

4) La importancia de promover que los métodos anticonceptivos que pueden tener efectos colaterales vayan siempre acompañados de una vigilancia médica. 
5) La importancia de avanzar en los programas de planificación familiar en las áreas rurales, en las zonas marginadas y en las áreas indígenas del país. También se mencionó el fortalecimiento e impulso en planificación familiar a las entidades federativas con mayor rezago socioeconómico, todo lo anterior con el fin de disminuir las inequidades regionales.

6) Se llamó la atención sobre por qué en el Issste no se han impulsado los programas de planificación familiar, cuando tienen un potencial muy grande por la enorme población afiliada.

7) También se destacaron dudas acerca de las cifras oficiales de población que se toman como base en los cálculos de las proyecciones de población.

8) Se preguntó si los datos sobre el método de oclusión tubaria debieran sumarse con los demás datos sobre uso de métodos anticonceptivos, ya que el primero es definitivo mientras los demás pueden no serlo.

9) Deberá promoverse mayor coordinación entre los sectores involucrados.

10) La necesidad de incrementar el presupuesto destinado a programas de planificación al reducirse la participación del sector externo.

\section{Sesión vespertina}

Los ponentes de la sesión vespertina fueron: Francisco Alba, de El Colegio de México, con el tema "Demografía mexicana e integración regional"; la doctora Ivonne Szasz, de la misma institución, quien trató el tema "Una visión integrada sobre salud reproductiva, situación de la mujer, participación en la fuerza de trabajo, educación y comunicación social", y el doctor Alfonso López Juárez, de MEXFAM, con el tema "El Programa Nacional de Población y su relación con las recomendaciones de la Conferencia de las Naciones Unidas sobre Población y Desarrollo de El Cairo".

\section{Ponentes}

El profesor Francisco Alba destacó la importancia del Programa Nacional de Población 1995-2000 dado que constituye una propuesta de 
acción a largo plazo, cuyo propósito es lograr la articulación entre población y desarrollo. Contrastó la propuesta del presente programa con los intentos anteriores realizados en la búsqueda de articulación población-desarrollo: el primero de ellos en los años trein ta y el segundo en los años setenta. La diferencia de la actual propuesta tiene que ver con el hecho de que los fenómenos demográficos en el México contemporáneo están enmarcados en el contexto de la globalización de la economía mundial. Éste es uno de los principales retos a los que -como sugiere Alba-, se enfrenta la actual política poblacional.

Los cambios en la economía mundial empujan a la población mexicana, según Alba, hacia adecuaciones fundamentales. Éstas tienen que ver con lo que él llama el reto de la capitalización de los recursos humanos, que se suma a otras demandas que pesan sobre el sistema económico y político del país.

El planteamiento de Alba adquiere importancia en el momento actual por la conveniencia de la integración de lo demográfico en la planeación del desarrollo. Según el autor, el proceso de globalización y la conformación de un bloque regional económico en América del Norte abre amplias posibilidades a la integración de la población mexicana a esos procesos económicos de naturaleza global.

La idea central manejada por Alba consiste en la necesidad de plantear una política poblacional que no sólo pretenda la armonización de la dinámica demográfica con la del desarrollo sino, además, armonizar las características de la población con la de los competidores y socios en el ámbito regional y global. La integración de la demografia mexicana con la economía norteamericana, se ha dado de manera espontánea por los flujos de trabajadores mexicanos hacia Estados Unidos. Ésta es sólo una parte de un proceso de integración mayor, el cual incluye las relaciones de México con Centroamérica. Hasta ahora, según Alba, la respuesta nacional parece calcar las del país vecino del norte. Las respuestas en el futuro deberían ser planteadas de manera distinta, ya que no obstante las características diversas de las tres sociedades y poblaciones, todas conforman un solo espacio regional.

Ivonne Szasz hizo una descripción de los contenidos del Programa Nacional de Población, destacando la seriedad del diagnóstico y la caracterización de la sociedad mexicana en que se sustenta el mencionado programa. Hizo hincapié en el reconocimiento, del cual parte el programa, de las desigualdades hombre-mujer como factor fundamental en la explicación de los comportamientos demográficos. 
No obstante, advierte la presencia de algunos problemas a lo largo del documento. Por ejemplo, subraya lo novedoso y audaz del análisis al incorporar elementos sociológicos fundamentales, mediante los cuales se presenta una imagen cruda y realista de la sociedad mexicana, pero critica la inconsistencia de la segunda parte del documento, la relacionada con las metas, en la cual se olvida el rigor inicial retomando planteamientos inscritos en viejos esquemas que no se adecúan a la problemática nacional.

Éste es el caso de las metas cuantitativas, las cuales muestran una voluntad de reducir la fecundidad, dejando intacto los problemas vinculados con las desigualdades hombre-mujer, la distribución del ingreso, la necesidad de la búsqueda de mejores niveles de vida, etc. Lo mismo ocurre en lo referente a la salud reproductiva, que parece ofrecer una simple visión antinatalista sin proponer servicios de anticoncepción integrados que incluyan la prevención de enfermedades de transmisión sexual, el tratamiento de la infertilidad, etcétera.

Otro ámbito de contradicciones en el PNP tiene que ver con el deseo por una parte de preservar costumbres, tradiciones y valores; y por otra, el planteamiento de la necesidad de un cambio de mentalidades. Este último no aparece definido en términos de sus propósitos y contenidos. Pero la principal tensión es la que tiene que ver con el contraste entre la aspiración de reducir las desigualdades de género y la formulación concreta del programa en materia de regulación de la fecundidad. No existe una mención concreta sobre aquellas relaciones de género que más afectan el comportamiento reproductivo; por ejemplo, las cuestiones ligadas al control de las mujeres sobre su propio cuerpo, la necesidad de separar las relaciones sexuales de la reproducción, la doble moral que inculca comportamientos diversos entre hombres y mujeres en materia sexual, y todos aquellos aspectos en los cuales se expresa el acceso diferencial al ejercicio de la sexualidad.

Finalmente, la doctora Szasz señala el hecho de que no se retoman los planteamientos iniciales sobre el combate a la pobreza como una cuestión fundamental para hacer frente a los problemas demográficos. Los:jóvenes no parecen tener un lugar fundamental en el programa, sobre todo, en el contexto de la composición etárea de la población mexicana.

A fin de lograr una mayor igualdad entre los géneros en materia de sexualidad, se mencionó la necesidad de formular medidas concretas para lograr la participación activa del varón en la anticoncep- 
ción y en la reproducción. Por último, se señaló la necesidad de plantear metas en número de usuarias satisfechas, de nuevos usuarios varones, de incorporación de jóvenes a los servicios, de diversidad de los métodos administrados, y de integración de metas anticonceptivas con metas de nuevos aceptantes de prevención de enfermedades de transmisión sexual, de tal manera que el programa se adecúe a los planteamientos formulados en el diagnóstico.

El doctor Alfonso López Juárez, después de señalar algunas semejanzas entre el Programa Nacional de Población y el Programa de Acción de la Conferencia de El Cairo, procedió a plantear algunos elementos críticos al PNP que sintetizó en cuatro puntos centrales:

- Cierta tibieza en el tratamiento del tema de la mujer. El documento del PNP parece plantear un análisis objetivo y crítico de la situación de la mujer en relación con la maternidad, fecundidad, participación económica y desigualdad que vive, no sólo respecto de la sociedad en general, sino frente al varón particularmente. No obstante, el documento elude la esfera del poder político, de la toma de decisiones, lo cual considera fundamental para revertir la situación de la mujer planteada en el diagnóstico.

- Omisión del concepto de salud sexual. El doctor López Juárez critica el carácter ideológico y reduccionista de la sexualidad al ser identificada con la reproducción. Plantea un salto cualitativo hacia la cultura de la salud sexual en donde se valorice la sexualidad como vehículo de comunicación, afecto y amor, independientemente de sus vínculos con la reproducción.

- Falta de prioridad hacia los jóvenes. El PNP reconoce la importancia que tiene el inicio temprano de la reproducción en términos de su impacto sobre la reproducción demográfica en general; parece extraño, sin embargo, que el documento no plantee una línea de política específica para losijóvenes. Por ello es necesario establecer el tránsito de la reproducción compulsiva a la nueva visión de la salud sexual, en la cual la sexualidad no aparece ligada a la reproducción.

- Una idealización de la familia mexicana. En este punto se realizó una crítica del concepto de familia utilizado en el PNP, según el cual, ésta aparece como un espacio de inculcación de valores, de superación familiar, de unidad nacional y ámbito de socialización que, entre otras cosas persigue la armonía. Lo que realmente existe en el ámbito familiar, es una reproducción del sistema autoritario, desigual y competitivo que existe en la sociedad en su conjunto. 


\section{Discusión general}

Los puntos más importantes discutidos en la sesión vespertina fueron los siguientes:

- Acotar las propuestas del Programa Nacional de Población al ámbito demográfico. No se le puede pedir a un programa demográfico que sustituya a uno de desarrollo, lo único que se le puede atribuir son las cuestiones que afectan e influyen en la dinámica de lo demográfico; no así las de política económica, de bienestar, de patrones culturales, etc., que a pesar de estar involucradas, son ámbitos de acción de otro tipo de política y no precisamente de la poblacional.

- Destacar la importancia de discutir sobre el tema del aborto, que no se ha incorporado en programas de población o de planificación familiar por problemas de tipo político.

- Reconocer que es un primer programa todavía solitario, en donde si bien su contexto es el Plan Nacional de Desarrollo, se requiere su integración a los otros programas económicos y sociales.

- Un punto de debate consistió en el análisis de las metas demográficas del Programa. Para algunos éstas se resuelven con un mayor énfasis en los contenidos de orden social del programa, más que en las metas cuantitativas. Por el contrario, otros destacan el carácter demográfico del Programa del cual surgirá poco a poco su contenido y material sociológico, así como político y de otras relaciones.

- Se debe reflexionar en términos del cumplimiento de las metas y del reto rural del Programa.

- Existe la preocupación respecto de las actuaciones de la Secretaría de Salud y del imss en cuanto a la práctica de los métodos anticonceptivos, sea el del dispositivo intrauterino o el de la oclusión tubaria sin consentimien to de la mujer. Entre los motivos por los cuales se da esta situación, se destaca el que la mujer no es siempre capaz de asumir ante su pareja la decisión de no procrear más y por lo tanto, responsabiliza al sector salud del hecho consumado.

- Un logro importante del Programa de Planificación Familiar en estos años, traducido en números, es el haber legitimado entre la población femenina mexicana el uso de medidas anticonceptivas. La investig ación -aún incipiente- sobre sexualidad indica que no existe conflicto moral entre las personas que usan anticonceptivos debido a sus creencias religiosas y convicciones. 
- En salud es fundamental un instrumento para el área rural y demás áreas marginadas constituido por tres acciones: nutrición, vacunación y salud reproductiva.

- Es muy importante la relación de la política de población de México con lo que pasa en Estados Unidos y Centroamérica. No se puede hacer política de población en México sin tomar en cuenta las situaciones migratorias entre estos países.

- La política de población no se puede dar de manera aislada en cada país sino que debe considerar las proximidades de algunos países y las relaciones específicas existentes entre ellos.

- La política de población no debe olvidar el contexto político; es más, el PNP se entiende como producto del consenso entre actores relevantes del aspecto político y fruto de la concertación.

- La Conferencia de El Cairo en realidad fue un parteaguas: lo principal es la ruptura entre la política de población concebida estrictamente en términos de su racionalidad demográfica, y la política de población que pone al servicio de la sociedad una serie de instrumentos para el ejercicio y disfrute pleno de los derechos reproductivos y humanos.

- Con respecto a la situación de la mujer, debe estar vinculada a los fenómenos demográficos y a la política de población como un todo, y éstos deberían abordarse en el marco del Programa Nacional de la Mujer. Existen dos aspectos básicos para el mejoramiento de la condición de la mujer: el tema relacionado con el acceso al poder y la toma de decisiones en los diferentes ámbitos. Hay que destacar la importancia que tiene la perspectiva de género en la conducción de una política.

- Se reconoció que algunos de los aspectos de género no son considerados dentro del Programa Nacional de Población.

- La política de población debe contar con ciertas características básicas como son: tener un carácter multisectorial y una vinculación con los programas de fecundidad, de planificación y salud reproductiva. Además, abordar la variedad de dimensiones para poder entender los problemas conceptuales de la definición de toda política de población.

- El PNP ha definido metas para el caso de la fecundidad y ha omitido una serie de metas cuantitativas o aun cualitativas para el resto de las variables demográficas. Esto es una limitación importante del Programa que tendrá necesariamente que ser corregida mediante los programas estatales donde otras metas podrían instrumentarse a nivel regional. 
- En cuanto al tema del aborto, no se trata de visualizarlo desde la perspectiva meramente demográfica, sino desde un punto de vista ético, moral, de percepciones y valores de la sociedad mexicana.

- Según el PNP, la migración en México, Estados Unidos y Centroamérica tiende a 0 hacia el año 2030 pero en realidad no significa que esté desapareciendo, sino que los flujos de una dirección se compensan con los de otra dirección.

\section{Bibliografia}

INEGI (1994), Encuesta Nacional de la Dinámica Demográfica, 1992. Metodología y tabulados, México.

Poder Ejecutivo Federal (1995), Programa nacional de población, 1995-2000, México, Consejo Nacional de Población. 
